UCRL-JC-121155 PREPRINT

\title{
Remote Monitoring of Emissions using On-vehicle Sensing and Vehicle to Roadside Communications
}

\author{
Donald T. Davis \\ Lawrence Livermore National Laboratory \\ Livermore, California \\ This paper was prepared for submittal to \\ 1995 SAE Future Tansportation Technology Conference and Exposition \\ Costa Mesa, CA \\ August 7-10, 1995
}

June, 1995

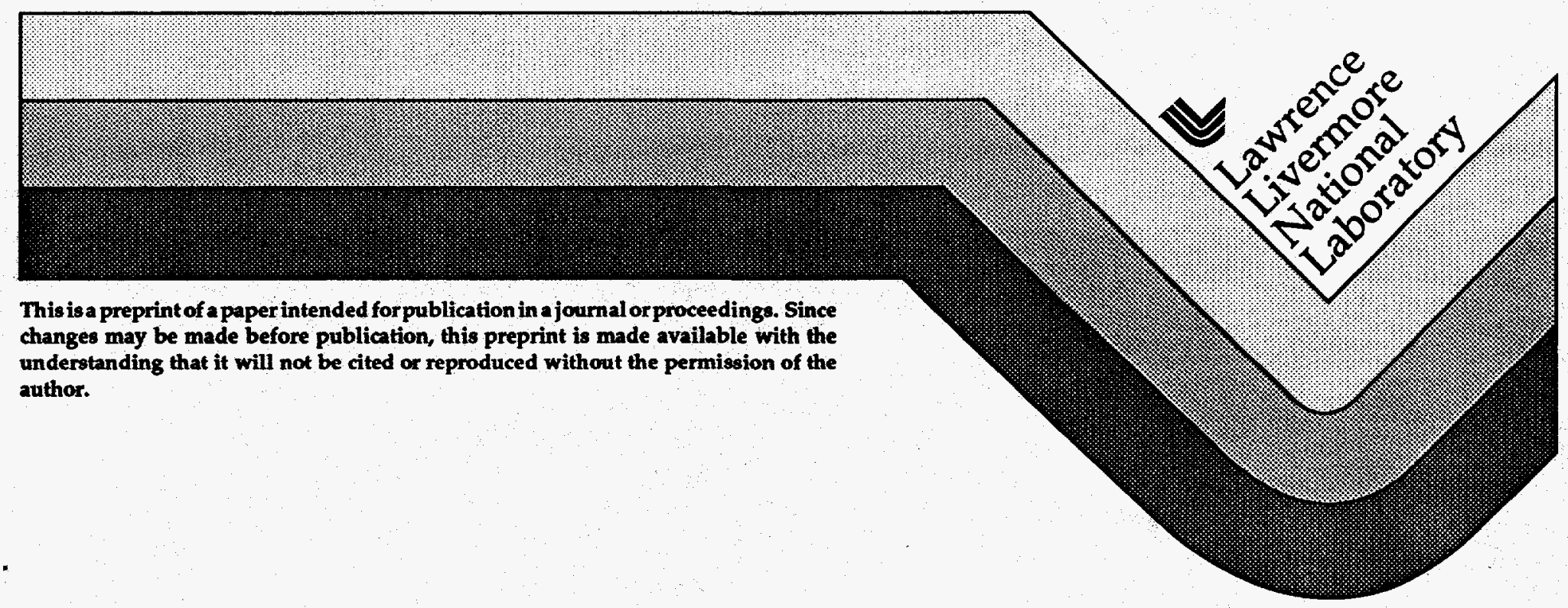




\section{DISCLAIMER}

This report was prepared as an account of work sponsored by an agency of the United States Government. Neither the United States Government nor any agency thereof, nor any of their employees, make any warranty, express or implied, or assumes any legal liability or responsibility for the accuracy, completeness, or usefulness of any information, apparatus, product, or process disclosed, or represents that its use would not infringe privately owned rights. Reference herein to any specific commercial product, process, or service by trade name, trademark, manufacturer, or otherwise does not necessarily constitute or imply its endorsement, recommendation, or favoring by the United States Government or any agency thereof. The views and opinions of authors expressed herein do not necessarily state or reflect those of the United States Government or any agency thereof. 


\section{DISCLAIMER}

Portions of this document may be illegible in electronic image products. Images are produced from the best available original document. 


\title{
"Remote Monitoring of Emissions using On-vehicle Sensing and Vehicle to Roadside Communications"
}

\author{
Donald T. Davis \\ Lawrence Livermore National Laboratory
}

\begin{abstract}
Recent developments in on-vehicle electronics makes practical remote monitoring of vehicle emissions compliance with CARB and EPA regulations. A system consisting of emission controls malfunction sensors, an on-board computer $(\mathrm{OBC})$, and vehicle-to-roadside communications (VRC) would enable enforcement officials to remotely and automatically detect vehicle out of - compliance status. Remole sensing could be accomplished at highway speeds as vehicles pass a roadside RF antenna and reader unit which would interrogate the on-vehicle monitoring and recording system.
\end{abstract}

This paper will focus on the hardware system components required to achieve this goal with special attention to the VRC; a key element for remote monitoring. This remote sensing concept piggybacks on the development of inexpensive VRC equipment for automatic vehicle identification for electronic toll collection and intelligent transportation applications.

Employing an RF transponder with appropriate interface to the OBC and malfunction sensors, a practical monitoring system can be developed with potentially important impact on air quality and enforcement. With such a system in place, the current - and costly and ineffective .- emission control strategy of periodic smog checking could be replaced or modified.

\section{INTRODUCTION}

The ability to remotely monitor a vehicle's compliance with emissions regulations in near real-time could result in better air quality and lower cost to the public. Two factors make this possibility very practical: 1) regulations which require the manufacturers to install equipment on the vehicle (starting in 1996) to detect and record significant out-of-compliance events and the ability to recover this data through a standard interface, and 2) inexpensive vehicle-to-roadside communication devices which can serve multiple applications.

Title 13 Section 1968.1 of the California Code of Regulations contains the malfunction and diagnostic requirements known as On-Boand Diagnostics II (OBD II). Similar Federal EPA regulations are contained in 40 CFR Part 86. Manufacturers must include monitoring sensors to detect the malfunction of vehicle emission control systems and record them in the vehicle's computer. The malfunction is also indicated on the instrument panel to warn the driver. Through the mandated electrical connector, information about the malfunctioning component(s) is made available to service technicians and smog checkers.

Unfortunately, one can not be sure that the vehicle owner will correct the problem when it first occurs rather than wait as long as the next bi-annual smog check. With remote monitoring, authorities could detect out-ofcompliance vehicles earty and take corrective action. In fact, with a system in place that could assure monitoring of all vehicles, the smog check program could be discontinued. The smog check program has been characterized as very expensive considering its impact (4 to $11 \%$ emissions reduction).

\section{PROPOSED REMOTE MONITORING SYSTEM}

Let us consider the elements of this proposed remote monitoring system. First are the on-board sensors, engine computer and digital network. Modern vehicles use on-board computers to carry out power train controls, and digital communication networks are anticipated (SAE J-1850). OBD II requires the monitoring of all emission control systems and all electronic components that can affect emissions when malfunctioning. It also requires the engine computer to record emission control fallures under reat-wortd driving conditions that result in emission levels above those allowable for the vehicle in question; tests not possible in 
the current smog check program. At this time, this information is only avallable to a repair lechnician using an OBD II Scan Tool (SAE J-1978) through a standard connector (SAE J-1962) and data format (SAE J-2012) when the owner brings it in for repair or certification.

The second element required for remole monitoring is a communication link with interface to the OBD II sensor data. Inexpensive and multipurpose Radio Frequency (RF) devices are starting to appear in vehicles which would suit remote monitoring very well. The FCC has recently issued permanent rules which allow such systems to operate in the $915 \mathrm{MHz}$ ISM band. Electronic Toll and Traffic Management (ETTM) on-vehicle transponder (tag) and roadside reader systems are finding important applications for short-range communications between moving vehicles and the roadside. The on-vehicle tags respond to inquiries from a roadside reader when passing through the reader's field of view; limited by design to a single vehicle for toll collection but capable of covering a multi-lane highway for remote monitoring. In the case of electronic toll collection, the tag transmits its account number when property requested to do so.

These systems operate typically at 300 to 500 kilobits per second (kbps) data rates so that up to several kilobits of data could be exchanged between the tag and reader while the vehicle passes through the reader's field of view; much more than required for transmission of the OBD II data. However, since the J-1850 network can not supply data at such a high rate, the lag must dedicate some of its memory to store the OBD II malfunction data and provide a proper interface to the J-bus. ETTM tags are currently available with adequate memory size for many applications and $\mathrm{J}-1850$ interfaces could be added very quickly and inexpensively.

ETTM systems are very flexible. An ETTM tag can be programmed to serve multiple functions with the same device (e.g. toll collection, commercial vehicle electronic clearance, remote emission monitoring and parking fees) each with differing message sets and communication protocol. This is achieved using dedicated tag memory sections for each application; and digital codes in the messages which define the function of the message, its data fields and the communication protocol.

What is needed for the remote monitoring application would be a message set consisting of: 1) a reader request for $\mathrm{OBD}$ II data, 2) a tag response incorporating the J-2012 OBD II data format and codes, and 3) a reader acknowledge message to silence the tag for a period of time.

\section{IN SEARCH OF AN ETTM NATIONAL STANDARD}

One important issue is the lack of a national standard for ETTM communications. Manufacturers typically have employed incompatible schemes which are now serving local or regional toll collection agencies. In an important standard setting action, the Lawrence Livermore National Laboratory helped the California Department of Transportation (Caltrans) develop a standard for ETTM systems in the state; the legislators had mandated interoperability between all electronic toll collection systems operating in California. This standard is included in Title 21 Chapter 16 of the California Code of Regulations. Appendix A contains a summary of key compatibility specifications. Subsequently, three companies have developed Title 21 compatible ETTM systems which they currently market world wide. Toll agencies in Kansas and Mexico have or are purchasing Title 21 compatible systems and other toll agencies are considering it.

Several other efforts are underway to try to establish a national standard. The ASTM has for some time now been stalemated by conflicting vendor interest in its efforts to reach a consensus on a national standard. The International Standards Organization (ISO) is working on a standard with major input from Europe (CEN). And, SAE has recently started a process for commercial vehicle automated electronic clearance (for weighstations and international border crossings) which is using as its draft position the Intelligent Transportation Society (ITS) of America ETTM Users Requirements developed by a set of toll collection agencies and the FHWA.

Regional toll agencies and the FHWA have so far been unable to bring about a national standard which would enable interoperability between systems from different vendors. CARB could be the agent that makes this possible. Vehicles that are designed to meet the California emission requirements tend to be sold in other states as well owing to the size of the California market and its leading position in smog reduction through CARB rule making. If CARB were to require ETTM tags in vehicles to implement remote monitoring then the standard selected might also be adapled by the EPA later country-wide. The Title 21 standard would be the obvious choice for CARB since the device could also be used for toll collection throughout the state (9 Caltrans bridges, the Golden Gate Bridge and new and anticipated toll roads) and other anticipated applications.

\section{REMOTE MONITOAING USING THE CALIFORNIA ETTM STANDARD}

Let us look at the Title 21 ETTM vehicle-to-roadside communications standard to see how we might use such a system to remotely monitor vehicle emission control maffunctions. The communications sequence used for toll collection, and a good model for the remote monitoring application, consists of a wake-up pulse and three messages. The wake-up pulse is the first signal which is sent by the roadside reader to the tag. This signal allows the tag to be normally in a stand-by mode to conserve its internal battery power and power-up the 
main circuits only during the short time it takes to exchange data with the reader. The three toll collection messages that follow the wake-up signal are contained in Appendix B. The first message is the polling message from the reader which in the case of toll collection asks the tag to respond with its ID (account) number. The tag recognizes the purpose of this message by the Transaction Record Type (TT) Code contained in the polling message. $8000 \mathrm{Hex}$ is a 16 bit code which serves to define the bits fields in the polling message and their function for toll collection. In the case of remote monitoring, one would specity another unique $\pi$ Code to request the tag to transmit the OBD II data. The second message is the tag's response to the polling message. Here the response message $\Pi$ Code is 0001 Hex which indicates that this message is a toll collection response and that the lag ID number is the data being transmitter by the tag. The third toll collection message is the acknowledge message. Here the $T$ code (CO0O HEX) identifies the message as an acknowledgment and the Transaction Status Code lets the tag know that a successful transaction has been achieved. After receiving a positive acknowledge message the tag will not respond to the same polling message for 10 seconds (letting other tags respond without interference).

Note the format of these three messages. Each message begins with a header consisting of an 8-bit selsyn to synchronize the receiver bit period to that of the transmitter, a 4-bit flag to indicate the start of message, a TT Code to define the message function and bit fields, the data fields and an error detection code ( CRC-16 or Cyclic Redundancy Code 16) which enables the receiver to check the accuracy of the received message.

In the case of our remote monitoring application, the same message format could be used but with different $\pi T$ Codes and message bit fields. That is, new $\pi$ codes would be selected and the data fields specified for each message. The main difference would be to use the data fields from the SAE J-2012 recommended practice in the response message from the tag. The polling message would let the tag know that the reader is looking for the emission control malfunction data rather than toll information. The data in the tag's OBD II memory section would then be transmitted to the reader.

Current ETTM tags contain a small battery and typically mount to the lower left hand side of the windshield using Velcro. If remote monitoring were to be required, the vehicle manufacturer may wish to install the tag elsewhere such as the back of the rear-view mirror with wiring to the J-1850 bus and vehicle battery. Current tags cost about $\$ 50$ to $\$ 75$ and are about the size of a cigarette pack. With greater volume as would be the case for remote monitoring of California vehicles, the cost and size could be substantially reduced.
SOME VOLUNTARY AND MANDATORY REMOTE MONITORING OPTIONS

A remote monitoring program could be started or operated as a voluntary program. In exchange for entering the program and installing a tag in the vehicle, the owner could be excused from the smog check program, a real incentive to many owners. System readers could be installed at key points on major highways and/or convenient in-town locations. The owner could be required to periodically drive by a reader, perhaps quarterly to annually, to stay in the program.

The numbers of readers required would be an important system cost consideration. Readers currently cost about $\$ 10 \mathrm{~K}$ but could be much less in greater volume as would be needed for coverage of an extended area remote monitoring program. One option is to employ the toll collection readers already planned by having them also collect the malfunction data; a good option for a voluntary program especially for those using the toll facilities. The data collected at reader sites could be downloaded nightly via telephone to a processing facility.

For a mandated program, the remote collection sites that make a bt of sense are refueling stations. Vehicles must refuel every week or so. This enables earty detection of malfunctions by enforcement officials and so the potential to minimize smog by tracking owner delays to repair. In addition, the owner might be offered an appointment at a nearby repair facility by the system computer. One disadvantage of using fueling stations is the number of readers required; an important element in the cost to implement such a system.

Since frequency of remote checking could be an issue, the time of last acknowledgment by an ETTM system could be recorded by the tag and made available to the owner through a display on his tag. He could press a button to see the date of his last interrogation, or a warning light could be lit if the mandatory interval is about to expire. Alternately, he could be notified by mail to drive by a reader as part of an annual check for vehicle licensing.

\section{PRIVACY}

Another important issue would be privacy. With distributed remote monitors it may be possible to learn something about the owner's movements that he may think is a potential invasion of his privacy. Assurances or regulations would have to be implemented so that such dala could not be used against the owner; there should be no law enforcement access to the data. The police should not be allowed by law to petition for the release of such data. Alternately, the location information could be suppressed; only the date of the most recent check and the first date of malfunction detection need be saved be the system computer. 
OBD II regulations mandate on-vehicle electronics to detect and record the malfunction of vehicle emission control components and systems. The data is stored in the engine's microprocessor memory in a standard format which is available through a standard electrical connector. Remote monitoring of the OBD II status is possible using ETTM RF tags interfaced to the vehicle's digital network, computer or OBD II connector, and roadside reader/interrogator units. Implementation of such a system depends on a number of factors including social issues, system technical issues, and vehicle and infrastructure costs. These factors must be weighed against potential benefits to vehicle owners who might be allowed to bypass the smog check program and society at large which might benefit from improved air quality. A costbenefit analysis of the various options available for using remote monitoring is required to determine the best approach.

\section{ACKNOWLEDGMENTS}

This work performed under the auspices of the U.S. Department of Energy by the Lawrence Livermore National Laboratory under Contract No. W-7405-ENG48.

\section{REFERENCES}

1) Davis, Donald T., 1993, "An Aluomatic Vehicle ID System for Toll Collecting", Lawrence Livermore Laboratory Report UCRL-TB-113409.

\section{AUTHOR BIOGRAPHY}

Dr. Davis received his BS, MS and PhD degrees in Electrical Engineering all from the University of Minnesota. Since 1965 he has worked at the Lawrence Livermore National laboratory; the last five years in the Transportation Technology Program were he currently is Associate Program Leader applying LLNL technologies to transportation problems. During this time he has also supported Caltrans and FHWA in the field of vehicle to roadside communications; developing communication protocols, designing ETTM hardware and pursuing national and international ETTM standards. His mailing address is Don T. Davis, LLNL, 7000 East Ave. L-644, Livermore CA, 94551 and phone number 510/422. 8801. 


\section{Summary of Key Compatibility Specifications}

\section{Reader speciflcations}

Wake-up signal

Reader send mode (downlink)

Carrier frequency

Carrier modulation

Data bit rate

Number of data bits

Field strength at transponder antenna $10^{\prime \prime} 1$ " bits followed by a $100 \mu$ s delay

$915 \pm 13 \mathrm{MHz}$

Unipolar ASK (Manchester encoded)

$300 \mathrm{kbps}$

Application specific

$500 \mathrm{mV} / \mathrm{m}$ (minimum)

\section{Transponder specifications}

Technology type

Transponder send mode (uplink)

Carrier frequency

Carrier modulation

Subcarrier modulation

Subcarrier frequencies

Data bit rate

Number of data bits

Wake-up (response) threshold

Transponder antenna

Polarization

Field-of-view

Location
Modulated backscatter

Same as reader send mode

Subcarrier AM

FSK

$600 \mathrm{kHz} \pm 10 \%$ and $1200 \mathrm{kHz} \pm 10 \%$ $300 \mathrm{kbps}$

Application specific

$500 \mathrm{mV} / \mathrm{m} \pm 10 \%$

\section{Horizontal}

Operation within $90^{\circ}$ conical angle in the forward direction

Front of vehicle 


\section{APPENDIX B}

\section{First Set of Caltrans-specified Polling, Transponder, and Acknowledge Message Formats}

Field definition

Polling message

Header code

Selsyn

Flag

Transaction record type code

Agency Code

Error detection code

Transponder message

Header code

Selsyn

Flag

Transaction record type code

Transponder ID number

Error detection code

Acknowledge message

Header code

Selsyn

Flag

Transaction record type code

Transponder ID code

Reader ID code

Transaction status code

Error detection code

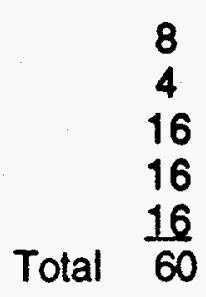

AA

$C$

8000

16

$\frac{16}{60}$

Hexadecimal valve

$\stackrel{\text { A }}{\mathrm{C}}$

32

16

Total $\frac{16}{76}$

8
4
16
32
32
16
16
Total $\quad \frac{124}{124}$

AA

C $\mathrm{COOO}$ 